\title{
Sleepless nights mean worse metabolism: a link to cardiovascular risk in older women
}

\author{
Karolina Piotrowicz ${ }^{1}$. Barbara Gryglewska ${ }^{1}$. Jerzy Gąsowski ${ }^{1}$
}

Published online: 13 August 2021

(c) European Geriatric Medicine Society 2021

Insomnia is one of the most common sleep disorders in old age [1]. Consisting of both nocturnal and daytime symptoms, insomnia can have a hugely detrimental effect on an individual's functionality and quality of life [2-4]. Moreover, insomnia has been associated with an increased risk of all-cause and cardiovascular mortality [5].

Chang et colleagues, in their cross-sectional study of 336 Taiwanese older adults, published in this issue of the EGM Journal, found that insomnia was associated with metabolic syndrome among older women but not men [6]. The presence of the association, after adjustment for confounders, between insomnia and metabolic syndrome in older women, points to the interesting possibility that sleep disorders could be a factor associated with increase of cardiovascular risk with aging of the female population. The pathophysiological links between a disrupted sleep-wake cycle, and the circadian rhythm as a whole, and metabolic dysregulation have been postulated. A lack of restorative sleep, as a consequence of insomnia, with short sleep duration, has been linked to oxidative stress, endothelial dysfunction, and inflammaging. Further, the functional disruption of the immune system and endocrine homeostasis leads to increased sympathetic nervous system activity and metabolic derangements that increase the burden of morbidity and mortality [7].

The results by Chang et al. are in line with the recently updated systematic review and meta-analysis on the association between sleep duration and metabolic syndrome [8]. As showed by Xie et al., based on the results of 36 crosssectional and 9 longitudinal studies, a short sleep duration was associated with a significantly higher risk of incidence

Jerzy Gąsowski

jerzy.gasowski@uj.edu.pl

1 Department of Internal Medicine and Gerontology, Jagiellonian University Medical College, University Hospital, 2 Jakubowskiego St., Building I, 5th floor, 30-688 Kraków, Poland
$(\mathrm{RR}=1.28,95 \%$ CI $1.07-1.53)$ and prevalence $(\mathrm{OR}=1.11$, 95\% CI 1.05-1.18) of metabolic syndrome in the general population [8]. Sleep disorders have gained a broad recognition as potent cardiometabolic risk factors. Recently, in a statement from the Italian Society of Hypertension, insomnia and sleep disorders in a wide context of cardiovascular comorbidities, acting through metabolic disorders, have been pointed as important risk factors for resistant hypertension [9]. On top of that, a consensus document and a joint statement on insomnia evaluation and management in clinical practice were released by the five Italian scientific societies (Italian Association of Sleep Medicine (AIMS), Italian Association for the Fight Against Stigma (AILAS), Italian Society of Consultation-Liaison Psychiatry (SIPC), Italian Society of Neuropsychopharmacology (SINF), and Italian Society of Psychosomatic Medicine (SIMP)) [10]. However, none of them are solely dedicated to older adults. When caring for older patients, some particularities must be considered. First, diagnostic vigilance for sleep-related problems in old age should be maintained. Awareness campaigns with widespread screening practices for sleep disorders in the elderly should be a priority. In a rapidly changing COVID-19 environment, some of the initial screening measures can be implemented with the support of telemedicine. Second, as part of good practices, comorbid conditions and medications should be carefully assessed when sleep disorders are diagnosed and treated, remembering that some sex differences were observed between sleep disturbances and multimorbidity [10-12]. As confirmed by Chang et al. [6], a meticulous evaluation of metabolic syndrome criteria, especially in older women, might be justified. Last but not least, an individual's lifestyle should be assessed and a non-pharmacological approach to not only insomnia management, but potential cardiovascular and metabolic comorbidities as well, should be introduced. 
Funding This research did not receive any specific grant from funding agencies in the public, commercial, or not-for-profit sectors.

\section{Declarations}

Conflict of interest None.

Ethical approval Not Applicable.

Informed Consent Not Applicable.

\section{References}

1. Patel D, Steinberg J, Patel P (2018) Insomnia in the elderly: a review. J Clin Sleep Med 14:1017-1024

2. Basta M, Simos P, Bertsias A, Duijker G, Zaganas I, Koutentaki E et al (2018) Association between insomnia symptoms and cognitive impairment in the Cretan Aging Cohort. Eur Geriatr Med 9:697-706

3. Endeshaw Y (2019) Self-reported insomnia symptom, sleep duration and the risk of recurrent falls among older men and women. Eur Geriatr Med 10:303-312

4. Pana A, Sourtzi P, Kalokairinou A, Pastroudis A, Chatzopoulos S-T, Velonaki VS (2021) Association between muscle strength and sleep quality and duration among middle-aged and older adults: a systematic review. Eur Geriatr Med 12:27-44

5. Ge L, Guyatt G, Tian J, Pan B, Chang Y, Chen Y et al (2019) Insomnia and risk of mortality from all-cause, cardiovascular disease, and cancer: systematic review and meta-analysis of prospective cohort studies. Sleep Med Rev 48:101215
6. Chang HC, Hsu YH, Chou MY, Chu CS, Su CS et al (2021) Insomnia in older adult females is highly associated with metabolic syndrome. Eur Geriatr Med. https://doi.org/10.1007/ s41999-021-00543-y

7. Nobre B, Rocha I, Morin CM, Cruz MME (2021) Insomnia and circadian misalignment: an underexplored interaction towards cardiometabolic risk. Sleep Sci 14:55-63

8. Xie J, Li Y, Zhang Y, Vgontzas AN, Basta M, Chen B et al (2021) Sleep duration and metabolic syndrome: an updated systematic review and meta-analysis. Sleep Med Rev 59:101451

9. Del Pinto R, Grassi G, Ferri C, Pengo MF, Lombardi C, Pucci G et al (2021) Diagnostic and therapeutic approach to sleep disorders, high blood pressure and cardiovascular diseases: a consensus document by the Italian Society of Hypertension (SIIA). High Blood Press Cardiovasc Prev 28:85-102

10. Palagini L, Manni R, Aguglia E, Amore M, Brugnoli R, Girardi P et al (2020) Expert opinions and consensus recommendations for the evaluation and management of insomnia in clinical practice: joint statements of five Italian Scientific Societies. Front Psychiatry 11:558

11. Capiau A, Foubert K, Somers A, Petrovic M (2021) Guidance for appropriate use of psychotropic drugs in older people. Eur Geriatr Med 12:577-583

12. Helbig AK, Stöckl D, Heier M, Thorand B, Schulz H, Peters A et al (2017) Relationship between sleep disturbances and multimorbidity among community-dwelling men and women aged 65-93 years: results from the KORA Age Study. Sleep Med $33: 151-159$

Publisher's Note Springer Nature remains neutral with regard to jurisdictional claims in published maps and institutional affiliations. 